\title{
Profile of a low-Mach-number shock in two-fluid plasma theory
}

\author{
M. Gedalin ${ }^{1}$, Y. Kushinsky ${ }^{1}$, and M. Balikhin ${ }^{2}$ \\ ${ }^{1}$ Department of Physics, Ben-Gurion University, Beer-Sheva, Israel \\ ${ }^{2}$ Department of Automatic Control and Systems Engineering, University of Sheffield, Sheffield, UK
}

Correspondence to: M. Gedalin (gedalin@bgu.ac.il)

Received: 9 March 2015 - Revised: 13 July 2015 - Accepted: 10 August 2015 - Published: 18 August 2015

\begin{abstract}
Magnetic profiles of low-Mach-number collisionless shocks in space plasmas are studied within the two-fluid plasma theory. Particular attention is given to the upstream magnetic oscillations generated at the ramp. By including weak resistive dissipation in the equations of motion for electrons and protons, the dependence of the upstream wave train features on the ratio of the dispersion length to the dissipative length is established quantitatively. The dependence of the oscillation amplitude and spatial damping scale on the shock normal angle $\theta$ is found.
\end{abstract}

Keywords. Interplanetary physics (interplanetary shocks; planetary bow shocks) - space plasma physics (shock waves)

\section{Introduction}

Collisionless shocks represent one of the most ubiquitous strong nonlinear phenomena in space plasmas. The main role of the collisionless shocks is to convert the energy of the directed flow into heating for the bulk of the plasma and into the acceleration of a fraction of the initial particle distribution to high energies. While the acceleration mechanism works mainly on large scales, the heating and the beginning of the acceleration occurs at the shock transition itself. Since the shock width is on the order of or smaller than the convective ion gyroradius (Greenstadt et al., 1975, 1980; Russell et al., 1982; Mellott and Greenstadt, 1984; Scudder et al., 1986; Mellott and Livesey, 1987; Farris et al., 1993; Hobara et al., 2010), the macroscopic electric and magnetic fields inside the transition layer govern the charged-particle motion there. Therefore, knowledge of the shock front structure is crucial for understanding the processes related to shocks. Observations show that, with the increase in the Mach number, the shock transition becomes progressively more com- plicated (Greenstadt et al., 1975, 1980; Russell et al., 1982; Mellott and Greenstadt, 1984; Scudder et al., 1986; Mellott and Livesey, 1987; Farris et al., 1993). In low-Mach-number shocks the transition region is known to be almost monotonic or accompanied by magnetic oscillations decaying upstream and downstream (Farris et al., 1993; Balikhin et al., 2008; Russell et al., 2009). Low-Mach-number shocks are subcritical or marginally critical collisionless shocks in magnetized plasmas (Kennel et al., 1985; Mellott, 1985), where the number of reflected ions is negligible. Although there is no theoretically established upper limit for the Mach number for such shocks, usually shocks with Alfvénic Mach numbers lower than 3 fall within this category (Mellott, 1985; Thomsen et al., 1985; Farris et al., 1993). Most of observed interplanetary shocks in the heliosphere are low-Mach-number shocks (Russell et al., 2009; Neugebauer, 2013). Many cosmological shocks, responsible for heating and acceleration, may be low-Mach-number shocks (Ryu et al., 2003; Hong et al., 2014).

So far, there has been no satisfactory theoretical description of the shock front even for low-Mach-number shocks. The magnetohydrodynamic (MHD) approach, which treats the plasma as a single conductive fluid, does not possess any characteristic spatial lengths and does not allow us to resolve the transition layer. If no dissipation is included, MHD provides only the relation between the upstream and downstream plasma parameters in the form of RankineHugoniot relations (RHs) (de Hoffmann and Teller, 1950). Derivation of the latter requires the introduction of additional assumptions, such as specifying the state equations for plasma species. RHs refer only to the asymptotic values of the plasma and magnetic field parameters, while the transition from one asymptotic value to the other remains unknown. Invoking dissipation in MHD, it becomes possible to describe a nonzero width transition. The typical way 
of including dissipation is to add resistivity to Ohm's law and/or add ad hoc viscosity terms to the equation of motion for the plasma. Yet, the width is determined by the dissipation alone and should be significantly larger than the ion inertial length for MHD to be valid (Kennel et al., 1985; Kennel, 1988). During the last decades supercritical quasi-perpendicular shocks (Bale et al., 2005; Krasnoselskikh et al., 2013) and quasi-parallel shocks (Burgess and Scholer, 2013) have attracted more attention because of their complicated pattern and the role of kinetic effects. Yet a theoretical description of the magnetic profile of even a lowMach-number shock remains a challenge. Observations show that the ramp width and the upstream oscillation wavelength is on the order of or less than the ion inertial length even in low-Mach-number shocks (Greenstadt et al., 1975, 1980; Mellott and Greenstadt, 1984; Farris et al., 1993). In this regime MHD breaks down and the two-fluid plasma theory (TFPT) has to be used (Krall and Trivelpiece, 1973). TFPT describes each species (electrons and protons) with the use of the continuity equation and Euler equation with the pressure and Lorenz force included. The plasma equations are coupled to the Maxwell equations via the charge and current densities produced by both species together. The pressure $p_{i j}$ is only the second moment of the distribution function $f(\boldsymbol{v})$, as follows: $p_{i j}=m \int\left(v_{i}-V_{i}\right)\left(v_{j}-V_{j}\right) f(\boldsymbol{v}) \mathrm{d} \boldsymbol{v}$, where $V_{i}$ is the bulk velocity (Krall and Trivelpiece, 1973). In the absence of collisions, the distribution function does not have to be isotropic so that the pressure is, in general, a tensor. Yet in many cases adopting the approximation of a scalar pressure $p_{i j}=p \delta_{i j}, \delta_{i j}$ being the Kronecker symbol, is widely accepted. TFPT equations are completed with the state equations $p(n)$ for each species (here $n$ is the number density). The polytropic law $p \propto n^{G}$ is widely used as a state equation. In addition to the pressure and Lorenz force, dissipative terms which describe phenomenologically the momentum exchange between the species phenomenologically can be added to the equations of motion (Krall and Trivelpiece, 1973). In a collisionless plasma this momentum exchange is usually related to anomalous resistivity (Sagdeev and Galeev, 1969; Papadopoulos, 2013).

Standard dissipation-free polytropic TFPT allows solitons and periodic nonlinear waves but does not allow solutions decaying to different asymptotical states (Sagdeev, 1966; Kennel and Sagdeev, 1967; Kennel et al., 1988; Hada et al., 1989; Gedalin, 1998). In fact, TFPT with scalar ion pressure is valid only in the upstream region, since ions begin to gyrate just behind the ramp, which results in non-gyrotropic distributions and in a non-scalar pressure (Gedalin and Zilbersher, 1995; Gedalin, 1996a, b, 1997; Balikhin et al., 2008; Ofman et al., 2009; Ofman and Gedalin, 2013). Before entering the ramp, however, ions are expected to remain gyrotropic. Numerical simulations of low-Mach-number, low- $\beta(\beta \lesssim 1)$ quasi-parallel shocks (Omidi et al., 1990) have shown that, apart from the effects due to backstreaming ions, their structure is not very different from those of quasi-perpendicular ones. The main additional feature is the appearance of the upstream whistler wave train. It should be mentioned, however, that the whistler wavelength rapidly decreases with the increase in the shock angle and will become smaller than the simulation cell size, unless the latter is on the order of the electron inertial length. Ignoring the kinetic effects at the first stage, it is reasonable to apply TFPT with a simple polytropic pressure law in the upstream region, up to the plasma entry to the ramp, for both quasi-perpendicular and quasiparallel geometries. In the present study we restrict ourselves to the upstream region of the shock. A shock-like profile cannot form without some dissipation (Gedalin, 1998). It is expected that such dissipation can be produced by microinstabilities (Papadopoulos, 2013). In the upstream region one can expect to have weak anomalous resistivity. Including resistive dissipation in a scalar polytropic TFPT, we describe the profiles of low-Mach-number shocks for a wide range of angles between the shock normal and the upstream magnetic field. Our approach is similar to that of Gurevich and Pitayevsky (1974), who treated a shock ramp as a discontinuity constantly generating large-amplitude waves propagating upstream. We assume that a finite width ramp is established which acts as a large-amplitude perturbation for the upstream region. Time stationary magnetic oscillations would damp with the distance from the ramp due to the dissipation.

\section{Basic equations}

We consider a one-dimensional stationary plasma within TFPT, where all variables depend only on the coordinate $x$ along the shock normal. With the increase in the Mach number the collisionless shock front is known to develop deviations from planarity and become nonstationary (see, e.g., Bale et al., 2005). Yet observations show that, in low-Machnumber shocks, these deviations are weak (Greenstadt et al., 1975; Kennel et al., 1984; Farris et al., 1993). Electrons are treated as a massless fluid. The ion and electron kinetic pressures are assumed to be scalar and polytropic state equations are used. The resistive dissipation is included as a friction term between the two fluids. Quasi-neutrality, $n_{i}=n_{e}=n$, is assumed, which is natural for the spatial and temporal scales under consideration. With these assumptions the equations take the following form (Krall and Trivelpiece, 1973):

$$
\begin{aligned}
& m_{i} v \partial_{x} \boldsymbol{v}_{i}=e \mathbf{E}+\frac{e}{c} \boldsymbol{v}_{i} \times \mathbf{B}-\hat{\boldsymbol{x}} \partial_{x} p_{i}-v\left(\boldsymbol{v}_{i}-\boldsymbol{v}_{e}\right), \\
& 0=-e \mathbf{E}-\frac{e}{c} \boldsymbol{v}_{e} \times \mathbf{B}-\hat{\boldsymbol{x}} \partial_{x} p_{e}-v\left(\boldsymbol{v}_{e}-\boldsymbol{v}_{i}\right), \\
& \hat{\boldsymbol{x}} \times \partial_{x} \mathbf{B}=\frac{4 \pi}{c} n e\left(\boldsymbol{v}_{i}-\boldsymbol{v}_{e}\right), \\
& n v=J=\mathrm{const}, \quad \mathbf{E}_{\perp}=\mathrm{const}, \quad B_{x}=\mathrm{const},
\end{aligned}
$$

where $\hat{\boldsymbol{x}}$ is the unity vector along the $x$ axis, $v=\boldsymbol{v} \cdot \hat{\boldsymbol{x}}$, and $\perp$ denotes components perpendicular to $\hat{\boldsymbol{x}}$. Here $\mathbf{E}$ and $\mathbf{B}$ are the total electric and magnetic field, respectively, $\boldsymbol{v}_{\mathrm{s}}$ is the 
bulk velocity of the species $s=e, i, n_{\mathrm{S}}$ is the number density of the species $s$, and $p_{\mathrm{s}}$ is the pressure of the species $s$. The momentum exchange (friction between the electrons and protons) is described by the term with $v$. We have also taken into account that $n_{i}=n_{e}$ means $v_{i}=v_{e}$. Summing the $x$ components of Eqs. (1) and (2) and taking into account Eq. (3), one gets (see, e.g., de Hoffmann and Teller, 1950; Krall and Trivelpiece, 1973; Scudder et al., 1986)

$n m_{i} v^{2}+\frac{B^{2}}{8 \pi}+p=$ const, $\quad p=p_{i}+p_{e}$.

For the perpendicular components one has (Gedalin, 1998)

$m_{i} v \partial_{x} \boldsymbol{v}_{i \perp}=e\left[\mathbf{E}_{\perp}+\frac{v}{c} \hat{\boldsymbol{x}} \times \mathbf{B}_{\perp}\right]+\frac{e B_{x}}{c} \boldsymbol{v}_{i \perp} \times \hat{\boldsymbol{x}}-v\left(\boldsymbol{v}_{i \perp}-\boldsymbol{v}_{e \perp}\right)$,

$0=-e\left[\mathbf{E}_{\perp}+\frac{v}{c} \hat{\boldsymbol{x}} \times \mathbf{B}_{\perp}\right]-\frac{e B_{x}}{c} \boldsymbol{v}_{e \perp} \times \hat{\boldsymbol{x}}+v\left(\boldsymbol{v}_{i \perp}-\boldsymbol{v}_{e \perp}\right)$,

$\hat{\boldsymbol{x}} \times \partial_{x} \mathbf{B}_{\perp}=\frac{4 \pi}{c} n e\left(\boldsymbol{v}_{i \perp}-\boldsymbol{v}_{e \perp}\right)$.

Here subscript $\perp$ denotes vectors perpendicular to $\hat{\boldsymbol{x}}$. Summing up Eqs. (6) and (7) and using Eq. (8), one gets

$\boldsymbol{v}_{i \perp}=\frac{B_{x} \mathbf{B}_{\perp}}{4 \pi m_{i} n v}+\boldsymbol{V}$

$\boldsymbol{v}_{e \perp}=\boldsymbol{v}_{i \perp}-\frac{c}{4 \pi n e} \hat{\boldsymbol{x}} \times \partial_{x} \mathbf{B}_{\perp}$,

where $\boldsymbol{V}$ is a constant vector which is determined by the conditions at a particular reference point. There is significant freedom in choosing the reference point and the conditions there. This freedom will be used later, after deriving the equations in the general form. Respectively, $\boldsymbol{V}$ is specified below in Eq. (19). Substituting all this into Eq. (6), one arrives at the following equation for the magnetic field (cf. Gedalin, 1998):

$$
\begin{aligned}
& \frac{B_{x}}{4 \pi n} \partial_{x} B_{y}-\frac{c v}{4 \pi n e} \partial_{x} B_{z}=e F_{y}-\frac{e v}{c} B_{z}\left(1-\frac{B_{x}^{2}}{4 \pi m_{i} n v^{2}}\right), \\
& \frac{B_{x}}{4 \pi n} \partial_{x} B_{z}+\frac{c v}{4 \pi n e} \partial_{x} B_{y}=e F_{z}+\frac{e v}{c} B_{y}\left(1-\frac{B_{x}^{2}}{4 \pi m_{i} n v^{2}}\right),
\end{aligned}
$$

$\boldsymbol{F}=\mathbf{E}_{\perp}+\frac{B_{x}}{c} \boldsymbol{V} \times \hat{\boldsymbol{x}}$.

These equations should be completed with $n v=J=$ const and

$n m_{i} v^{2}+\frac{B_{y}^{2}+B_{z}^{2}}{8 \pi}+p(n)=Q=$ const.
The stationary points of Eqs. (11-12) are the points where $\partial_{x} B_{y}=\partial_{x} B_{z}=0$, that is,

$0=e F_{y}-\frac{e v_{0}}{c} B_{z 0}\left(1-\frac{B_{x}^{2}}{4 \pi m_{i} n_{0} v_{0}^{2}}\right)$,
$0=e F_{z}+\frac{e v_{0}}{c} B_{y 0}\left(1-\frac{B_{x}^{2}}{4 \pi m_{i} n_{0} v_{0}^{2}}\right)$,

where subscript 0 denotes values at (one of) the stationary point(s). Using the freedom in choosing the coordinate axes, we put $B_{y 0}=0$ and introduce the angle $\theta$ between the magnetic field at the stationary point and the normal, so that $B_{z 0}=B_{0} \sin \theta$ and $B_{x}=B_{0} \cos \theta$. Respectively, one has $F_{z}=0$ and

$F_{y}=\frac{v_{0}}{c} B_{0} \sin \theta\left(1-\frac{B_{0}^{2} \cos ^{2} \theta}{4 \pi m_{i} n_{0} v_{0}^{2}}\right)$.

For the ion velocities at the stationary point we find

$v_{i y 0}=V_{y}, \quad v_{z 0}=\frac{B_{0} \sin \theta \cos \theta}{4 \pi m_{i} n_{0} v_{0}}+V_{z}$.

Choosing the reference frame in which $\boldsymbol{v}_{i \perp 0}=0$ (this is the well-known normal incidence frame) sets

$V_{y}=0, \quad V_{z}=-\frac{B_{0} \sin \theta \cos \theta}{4 \pi m_{i} n_{0} v_{0}}$.

Using Eq. (6) one obtains $E_{z}=0$ and

$E_{y}=\frac{v_{0}}{c} B_{0} \sin \theta$.

It is natural to define the Alfvén velocity and the Alfvénic Mach number at the stationary point as

$v_{A}^{2}=\frac{B_{0}^{2}}{4 \pi m_{i} n_{0}}, \quad M=\frac{v_{0}}{v_{A}}$.

In order to achieve a better physical understanding, we introduce the following normalized variables:

$\boldsymbol{b}=\frac{\mathbf{B}_{\perp}}{B_{0} \sin \theta}, \quad V=\frac{v}{v_{0}}, \quad N=\frac{n}{n_{0}}=\frac{1}{V}$.

Then the derived equations take the following shape:

$l_{\mathrm{w}} \partial_{x} b_{y}-l_{\mathrm{d}} \partial_{x} b_{z}=N(1-s)-(1-N s) b_{z}$,

$l_{\mathrm{w}} \partial_{x} b_{z}+l_{\mathrm{d}} \partial_{x} b_{y}=(1-N s) b_{y}$,

$\frac{1}{N}+y b^{2}+x f(N)=1+y+x$.

Here

$s=\frac{\cos ^{2} \theta}{M^{2}}, \quad y=\frac{\sin ^{2} \theta}{2 M^{2}}, \quad x=\frac{\beta}{2 M^{2}}$,

$\beta=\frac{8 \pi p_{0}}{B_{0}^{2}}, \quad f(N)=\frac{p(N)}{p_{0}}$,

and the dispersion and dissipative length are

$l_{\mathrm{w}}=\frac{c B_{0} \cos \theta}{4 \pi n_{0} e v_{0}}=\frac{c \cos \theta}{M \omega_{p i}}, \quad l_{\mathrm{d}}=\frac{c^{2} v}{4 \pi n_{0} e^{2} v_{0}}=\frac{c^{2} \eta}{4 \pi v_{0}}$, 
where $\omega_{p i}^{2}=4 \pi n_{0} e^{2} / m_{i}$ and $\eta$ is the resistivity. The length $l_{\mathrm{w}}$ is easily recognizable as the inverse wave number of a low-frequency whistler wave standing in the frame which is moving with the velocity $v_{0}: v_{0}=\omega / k=$ $k c \cos \theta B_{0} / 4 \pi n_{0} e$ (Krall and Trivelpiece, 1973). Hereafter, $l_{\mathrm{w}}$ is referred to as "whistler wavelength". It is worth mentioning that $l_{\mathrm{d}}$ may depend on $\theta$ and $M$ only implicitly, via a possible dependence on $v$. At the same time, $l_{\mathrm{w}}$ rapidly drops with the increase in $M$ and/or $\theta$.

The nature of the stationary point is determined by the linearized equations

$l_{\mathrm{w}} \partial_{x} \delta b_{y}-l_{\mathrm{d}} \partial_{x} \delta b_{z}=\delta N-(1-s) \delta b_{z}$,

$l_{\mathrm{w}} \partial_{x} \delta b_{z}+l_{\mathrm{d}} \partial_{x} \delta b_{y}=(1-s) \delta b_{y}$,

$-\delta N+2 y \delta b_{z}+G x \delta N=0$,

where $G=\left(1 / p_{0}\right)(\mathrm{d} p / \mathrm{d} N)_{N=1}$ so that $v_{s}^{2}=G \beta v_{A}^{2} / 2$ is the local sound speed at the reference point. Assuming $\delta b_{y}, \delta b_{z}, \delta N, \delta V \propto \exp (k x)$, one has

$$
\begin{aligned}
& k^{2}\left(l_{\mathrm{w}}^{2}+l_{\mathrm{d}}^{2}\right)-C_{1} k l_{\mathrm{d}}+C_{2}=0, \\
& k_{1,2}=\frac{C_{1} l_{\mathrm{d}} \pm \sqrt{C_{1}^{2} l_{\mathrm{d}}^{2}-4 C_{2}\left(l_{\mathrm{w}}^{2}+l_{\mathrm{d}}^{2}\right)}}{2\left(l_{\mathrm{w}}^{2}+l_{\mathrm{d}}^{2}\right)}, \\
& C_{1}=\frac{\left(v^{2}-v_{s}^{2}\right)\left(v^{2}-v_{\mathrm{I}}^{2}\right)+\left(v^{2}-v_{\mathrm{F}}^{2}\right)\left(v^{2}-v_{\mathrm{SL}}^{2}\right)}{v^{2}\left(v^{2}-v_{s}^{2}\right)}, \\
& C_{2}=\frac{\left(v^{2}-v_{\mathrm{SL}}^{2}\right)\left(v^{2}-v_{\mathrm{I}}^{2}\right)\left(v^{2}-v_{\mathrm{F}}^{2}\right)}{v^{4}\left(v^{2}-v_{s}^{2}\right)},
\end{aligned}
$$

where $v_{\mathrm{I}}, v_{\mathrm{SL}}$, and $v_{\mathrm{F}}$ are the usual velocities of intermediate, slow, and fast MHD waves, respectively (Krall and Trivelpiece, 1973), defined locally at the stationary point:

$v_{\mathrm{I}}^{2}=v_{A}^{2} \cos ^{2} \theta$,

$v_{\mathrm{F}}^{2}=\frac{1}{2}\left[v_{A}^{2}+v_{s}^{2}+\sqrt{\left(v_{A}^{2}+v_{S}^{2}\right)^{2}-4 v_{A}^{2} v_{S}^{2} \cos ^{2} \theta}\right]$,

$v_{\mathrm{SL}}^{2}=\frac{1}{2}\left[v_{A}^{2}+v_{s}^{2}-\sqrt{\left(v_{A}^{2}+v_{s}^{2}\right)^{2}-4 v_{A}^{2} v_{s}^{2} \cos ^{2} \theta}\right]$.

Stationary points of the autonomous system of two first-order ordinary differential equations are classified according to the exponents $k_{1}$ and $k_{2}$ (see, e.g., Arnold, 1978). If $C_{2}<0$, then both $k_{1}$ and $k_{2}$ are real and $k_{1} k_{2}<0$, that is, the stationary point is a saddle point, which means that one special solution ends at the stationary point and another special solution starts at this point, while all other solutions do not arrive at this point at all. If $C_{2}>0$ and $D=C_{1}^{2} l_{\mathrm{d}}^{2}-4 C_{2}\left(l_{\mathrm{w}}^{2}+l_{\mathrm{d}}^{2}\right)>0$, then the stationary point is a node, which means that either all solutions end there or start there and the magnetic field vector rotates by a finite angle only. If $C_{2}>0$ and $D<0$, the stationary point is a focus, which is similar to the node, with the only difference being that the angle of the magnetic field rotation is infinite.
In order to have a shock solution with different upstream $(x \rightarrow-\infty)$ and downstream $(x \rightarrow \infty)$ asymptotic states, we need the magnetic perturbation, caused by the ramp, to decay toward $\pm \infty$. Thus, $\operatorname{Re} k_{1}>0$ and $\operatorname{Re} k_{2}>0$ are required at the upstream stationary point, and $\operatorname{Re} k_{1}<0$ and $\operatorname{Re} k_{2}<0$ are required at the downstream stationary point. For a fast magnetosonic shock in the upstream asymptotic state at $x \rightarrow-\infty$, one has $v>v_{\mathrm{F}}$, which means that both $C_{1}$ and $C_{2}$ are positive. This point is a node for strong dissipation, $D>0$, and a focus for weak dissipation, $D<0$. In both cases $\operatorname{Re} k_{1}>0$ and $\operatorname{Re} k_{2}>0$ are ensured by the presence of the dissipation. In the downstream asymptotic state $(x \rightarrow \infty)$, the evolutionarity conditions require that the velocity should be in the range $v_{\mathrm{I}}<v<v_{\mathrm{F}}$. If $v_{s}<v_{\mathrm{I}}$, the asymptotic point is a saddle and $\operatorname{Re} k_{1} \operatorname{Re} k_{2}<0$. If $v_{s}>v_{\text {I }}$ and $v<v_{s}$, the asymptotic point is a node. Since in this case $C_{1}>0$, the exponents $\operatorname{Re} k_{1}>0$ and $\operatorname{Re} k_{2}>0$ and magnetic perturbations do not damp toward $x \rightarrow \infty$. Thus, a resistive dissipation does not allow a fast shock solution for any Mach number.

\section{Upstream region}

As mentioned above, one cannot really expect that the simple scalar pressure TFPT be applicable behind the ramp. Indeed, the gyration of the ion distribution as a whole breaks down these approximations. The objectives of the present paper do not include the analysis of a non-gyrotropic pressure. However, the region upstream of the ramp should be well approximated by the approach adopted for sufficiently low Mach numbers when ion reflection in negligible. Moreover, we have shown that in an asymptotically super-fast magnetosonic flow, magnetic perturbation damps toward $x \rightarrow-\infty$. From

$\frac{\mathrm{d} N}{\mathrm{~d} b^{2}}=\frac{y N^{2}}{G x N^{G+1}-1}$,

the maximum achievable density is $N_{c}=(G x)^{-1 /(G+1)}$, which corresponds to the maximum possible

$b_{c}^{2}=\frac{1}{y}\left[1+y+x-\frac{G+1}{G N_{c}}\right]$,

with the maximum magnetic compression of $B_{t} / B_{0}=$ $\sqrt{\cos ^{2} \theta+b_{c}^{2} \sin ^{2} \theta}$.

We could not solve Eqs. (23-25) analytically. For numerical visualization below we have chosen the fast magnetosonic Mach number to be fixed at $M_{\mathrm{F}}=2$, while $\theta$ and $\beta$ are varied. A polytropic pressure is chosen, $p(N)=N^{G}$, with $G=5 / 3$ so that $v_{s}^{2} / v_{A}^{2}=G \beta / 2$. The Alfvénic Mach number is $M=M_{\mathrm{F}} v_{\mathrm{F}} / v_{A}$ and also varies with $\theta$ and $\beta$. The ratio of the dissipation and dispersion lengths in terms of resistivity is

$\epsilon=\frac{l_{\mathrm{d}}}{l_{\mathrm{w}}}=\left(\frac{\omega_{p i} \eta}{\cos \theta}\right)\left(\frac{\omega_{p i}}{\Omega_{i}}\right)=\left(\frac{\omega_{p i} \eta}{\cos \theta}\right)\left(\frac{c}{v_{A}}\right)=\left(\frac{M \omega_{p i} \eta}{\cos \theta}\right)\left(\frac{c}{v_{0}}\right)$. 

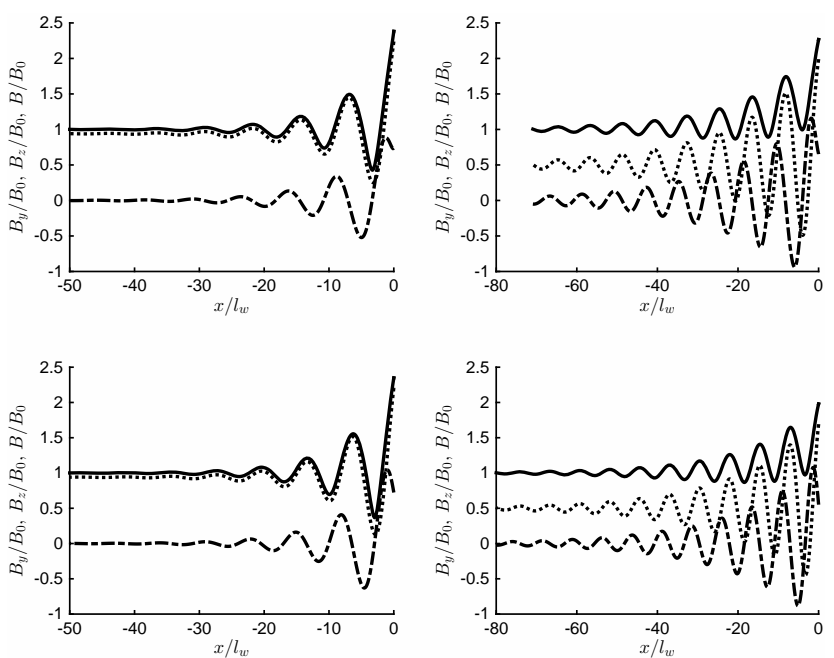

Figure 1. The total magnetic field (solid line) and the two perpendicular components, $B_{z} / B_{0}$ (dotted) and $B_{y} / B_{0}$ (dash-dotted) for the four cases: 1) $\theta=70^{\circ}, \beta=0.2, M=2.14$ (top left); 2) $\theta=30^{\circ}$, $\beta=0.2, M=2.05$ (top right); 3) $\theta=70^{\circ}, \beta=1.2, M=2.79$ (bottom left); and 4) $\theta=30^{\circ}, \beta=1.2, M=2.14$ (bottom right). In all cases the fast Mach number $M_{\mathrm{F}}=2$.

For the solar wind conditions, $v_{0} / c \sim 10^{-3}$, one would have $\eta \omega_{p i} \sim 10^{-3} \epsilon(\cos \theta / M)$. For the visualization below we have chosen $\epsilon=0.05 / \cos \theta$, which gives an approximate idea of the dependence of $\epsilon$ on the shock angle for constant resistivity. For the chosen parameters the latter is in the range $\eta \sim\left(10^{-5}-10^{-4}\right) \omega_{p i}^{-1}$.

Figure 1 compares the profiles obtained for the following four cases: 1) $\theta=70^{\circ}, \beta=0.2$ (top left); 2) $\theta=30^{\circ}$, $\beta=0.2$ (top right); 3) $\theta=70^{\circ}, \beta=1.2$ (bottom left); and 4) $\theta=30^{\circ}, \beta=1.2$ (bottom right). Coordinate $x$ is measured in the whistler wavelengths $l_{\mathrm{w}}$. The calculated Alfvénic Mach numbers are given in the figure caption. The profiles are rather similar. Longer wave trains for smaller $\theta$ are due to lower $\epsilon$.

In the quasi-perpendicular case, $\theta=70^{\circ}$, one of the perpendicular components of the magnetic field, $B_{y}$, always remains substantially smaller than the other, $B_{z}$, so that the polarization of the wave train is close to linear. In the quasiparallel case, $\theta=30^{\circ}$, the two components are comparable, and the polarization is elliptical, approaching a circular polarization. High- $\beta$ cases should be treated with caution since ion reflection may be noticeable even at low Mach numbers. Figure 2 shows the magnetic field profiles (total magnetic field) for all four cases. The wavelengths for $\theta=70^{\circ}$ are smaller than the wavelengths for $\theta=30^{\circ}$. For $\theta=30^{\circ}$ the wavelength is smaller for lower $\beta$.

In the above figures the coordinate is measured in whistler wavelengths $l_{\mathrm{w}}=c \cos \theta / M \omega_{p i}$. When keeping $M_{\mathrm{F}}$ and $\beta$ constant, the whistler wavelength rapidly decreases with the increase in $\theta$. Our analysis is done using the massless electron approximation and can be valid only if $l_{\mathrm{w}} \gg c / \omega_{p e}$, that

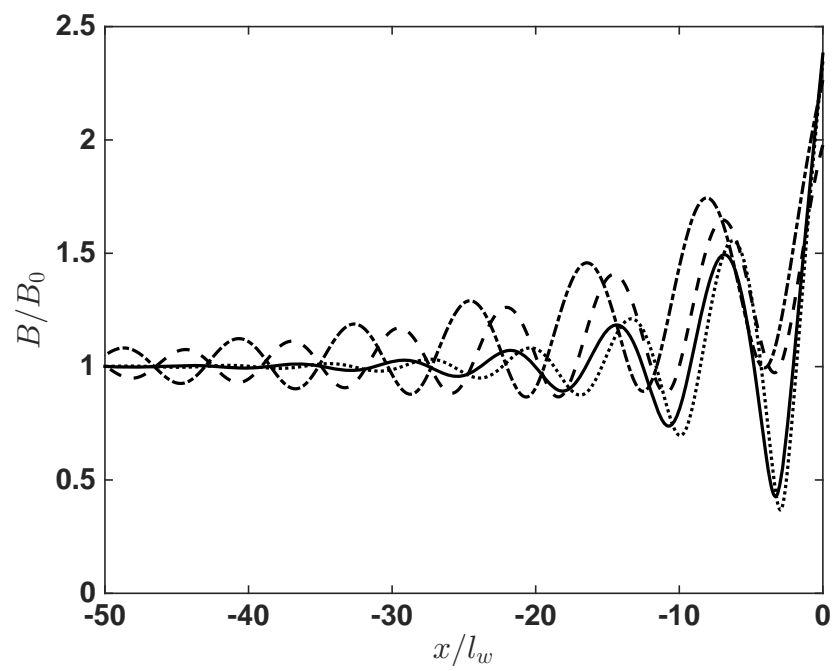

Figure 2. Total magnetic field for the four cases: (1) $\theta=70^{\circ}, \beta=$ 0.2 (solid line); (2) $\theta=30^{\circ}, \beta=0.2$ (dash-dotted line); (3) $\theta=$ $70^{\circ}, \beta=1.2$ (dotted line); and (4) $\theta=30^{\circ}, \beta=1.2$ (dashed line).

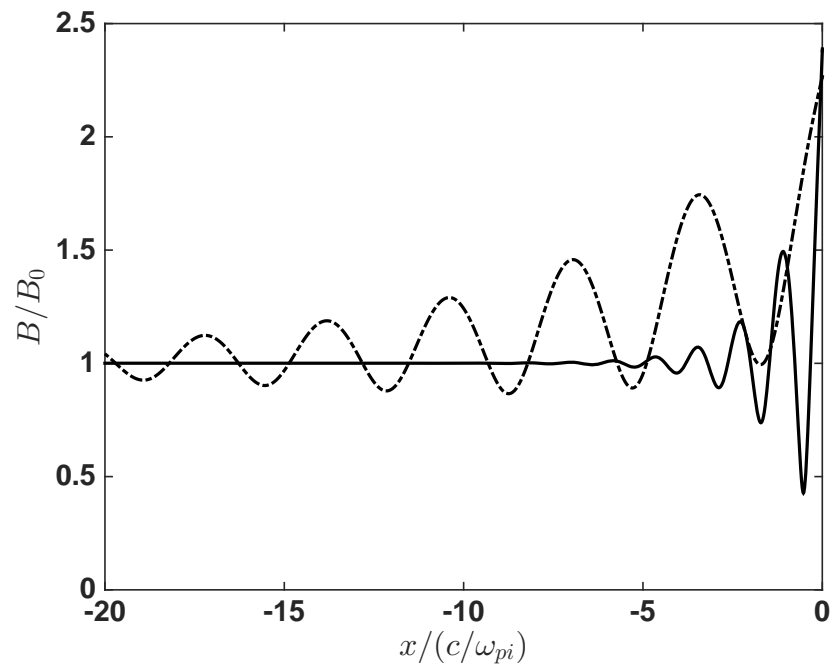

Figure 3. Comparison of the profiles for $\theta=70^{\circ}$ (solid line) and $\theta=30^{\circ}$ (dash-dotted line). Here the coordinate is measured in ion inertial lengths.

is, for $\cos \theta / M \gg\left(m_{e} / m_{i}\right)^{1 / 2}$. Figure 3 compares the profiles for $\theta=70^{\circ}$ and $\theta=30^{\circ}$ when the coordinate is measured in ion inertial lengths $c / \omega_{p i}$.

The representative values of $\theta$ and $\beta$ were chosen to illustrate the differences. Figures 4 and 5 show the profiles with additional sets of parameters.

Figure 4 shows the magnetic profiles for $M_{\mathrm{F}}=2$ and $\beta=0.2$ and four values of the angle between the shock normal and the upstream magnetic field $\left(\theta=15^{\circ}, 45^{\circ}, 60^{\circ}, 85^{\circ}\right)$. The number of oscillations drops rapidly with the increase in the angle. For the nearly perpendicular shock, $\theta=85^{\circ}$, the upstream wave train reduces to a magnetic dip just ahead of 

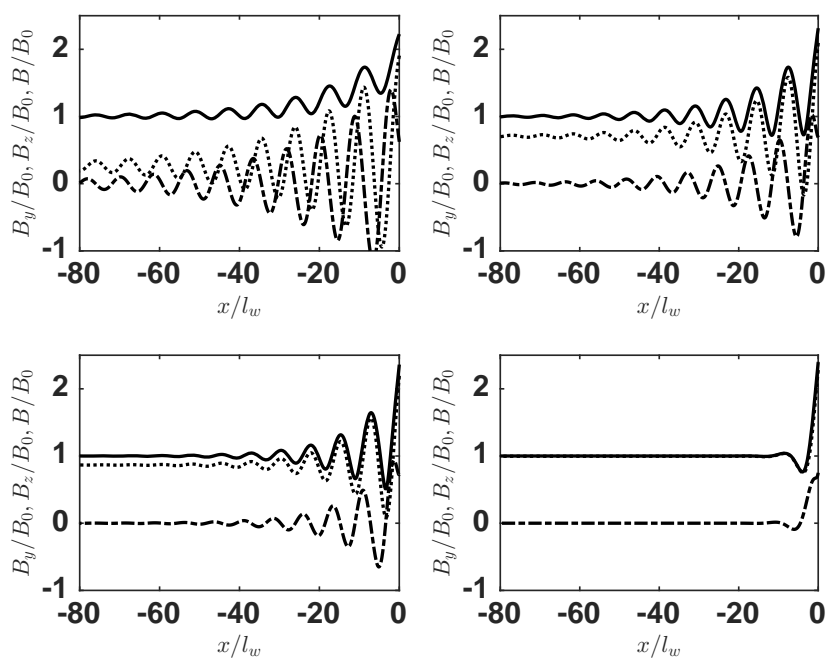

Figure 4. The total magnetic field (solid line) and the two perpendicular components, $B_{z} / B_{0}$ (dotted) and $B_{y} / B_{0}$ (dash-dotted). Top left: $\theta=15^{\circ} ; M=2.01$. Top right: $\theta=45^{\circ} ; M=2.09$. Bottom left: $\theta=60^{\circ} ; M=2.13$. Bottom right: $\theta=85^{\circ} ; M=2.16$. Other parameters: $\beta=0.2 ; M_{\mathrm{F}}=2$.
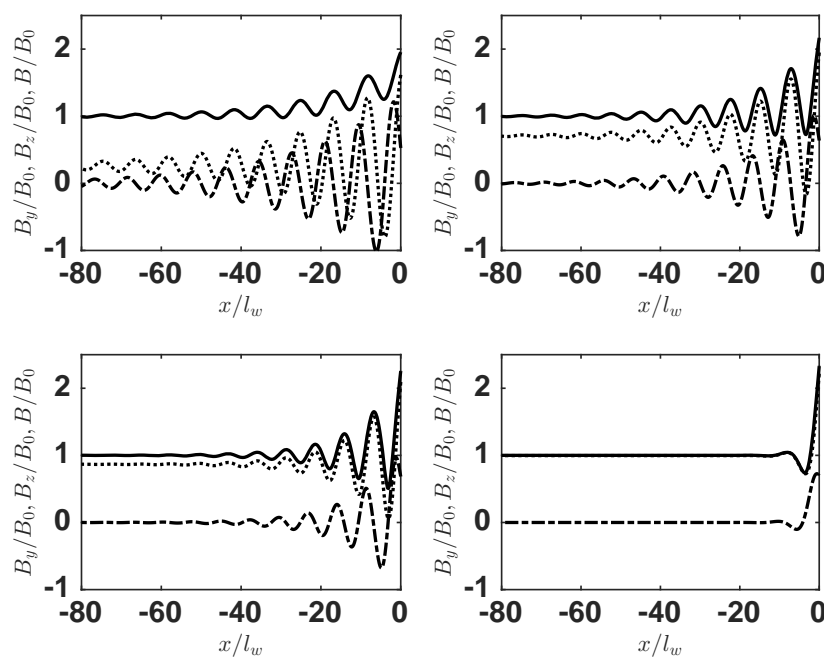

Figure 5. The total magnetic field (solid line) and the two perpendicular components, $B_{z} / B_{0}$ (dotted) and $B_{y} / B_{0}$ (dash-dotted). Top left: $\theta=15^{\circ} ; M=2.04$. Top right: $\theta=45^{\circ} ; M=2.24$. Bottom left: $\theta=60^{\circ} ; M=2.31$. Bottom right: $\theta=85^{\circ} ; M=2.38$. Other parameters: $\beta=0.5 ; M_{\mathrm{F}}=2$.

the ramp. In Fig. 5 the upstream plasma is hotter, $\beta=0.5$, while $M_{\mathrm{F}}$ and $\theta$ are the same as in Fig. 4. There is little difference between the behavior of the magnetic profiles for the same $M_{\mathrm{F}}$ and $\theta$ and different $\beta$. However, with the increase in $\beta$ for given $M_{\mathrm{F}}$ and $\theta$, the Alfvénic Mach number is higher. The whistler wavelength is smaller. Therefore, when measured in the ion inertial lengths, the profiles with higher $\beta$ will look "tighter" (compare with Fig. 2).

\section{Conclusions}

Simple TFPT with polytropic pressure and resistive dissipation is able to reproduce quantitatively the basic features of the upstream side of a low-Mach-number collisionless shock in a wide range of $\theta$ and $\beta$. In the dimensionless variables, the profiles are rather similar for constant $M_{\mathrm{F}}$, with only a weak dependence on $\theta$ and $\beta$. The length of the whistler wave train depends on the ratio between the dissipation and whistler wavelengths and rapidly decreases with the increase in this ratio. This similarity should not be surprising since the kinetic effects are negligible for low Mach numbers in the upstream region. Unless $\theta$ is too small, the species motion is governed by the magnetic field. The approximation of massless electrons is valid if $\cos \theta / M \gg\left(m_{e} / m_{i}\right)^{1 / 2}$. Thus, in a wide range of angles the upstream parts of low-Machnumber shocks should be similar. Observations would not show this similarity as long as the measurements are normalized not with the whistler wavelength but with the ion inertial length, as is widely accepted. The whistler wavelength rapidly decreases with the increase in the angle, while the ratio of the dissipation-to-whistler wavelength increases. In the quasi-perpendicular case the shock front should be much narrower and exhibit fewer oscillations.

Acknowledgements. M. Gedalin was partly supported by the Israel Science Foundation (grant No. 368/14).

The topical editor G. Balasis thanks two anonymous referees for help in evaluating this paper.

\section{References}

Arnold, V. I.: Ordinary differential equations, MIT Press, Cambridge, MA, USA, 1978.

Bale, S. D., Balikhin, M. A., Horbury, T. S., Krasnoselskikh, V. V., Kucharek, H., Möbius, E., Walker, S. N., Balogh, A., Burgess, D., Lembège, B., Lucek, E. A., Scholer, M., Schwartz, S. J., and Thomsen, M. F.: Quasi-perpendicular shock structure and processes, Space Sci. Rev., 118, 161-203, 2005.

Balikhin, M. A., Zhang, T. L., Gedalin, M., Ganushkina, N. Y., and Pope, S. A.: Venus Express observes a new type of shock with pure kinematic relaxation, Geophys. Res. Lett., 35, L01103, doi:10.1029/2007GL032495, 2008.

Burgess, D. and Scholer, M.: Microphysics of Quasi-parallel Shocks in Collisionless Plasmas, Space Sci. Rev., 178, 513-533, 2013.

de Hoffmann, F. and Teller, E.: Magneto-hydrodynamic shocks, Phys. Rev., 80, 692-703, 1950.

Farris, M., Russell, C., and Thomsen, M.: Magnetic structure of the low beta, quasi-perpendicular shock, J. Geophys. Res., 98, 15285-15294, 1993.

Gedalin, M.: Noncoplanar magnetic field in the collisionless shock front, J. Geophys. Res., 101, 11153-11156, 1996a.

Gedalin, M.: Transmitted ions and ion heating in nearly perpendicular low-Mach number shocks, J. Geophys. Res., 101, 15569$15578,1996 \mathrm{~b}$. 
Gedalin, M.: Ion heating in oblique low-Mach number shocks, Geophys. Res. Lett., 24, 2511-2514, 1997.

Gedalin, M.: Low-frequency nonlinear stationary waves and fast shocks: Hydrodynamical description, Phys. Plasmas, 5, 127132, 1998.

Gedalin, M. and Zilbersher, D.: Non-diagonal ion pressure in nearly-perpendicular collisionless shocks, Geophys. Res. Lett., 22, 3279-3282, 1995.

Greenstadt, E. W., Scarf, F. L., Russell, C. T., Formisano, V., and Neugebauer, M.: Structure of the quasi-perpendicular laminar bow shock, J. Geophys. Res., 80, 502-514, 1975.

Greenstadt, E. W., Scarf, F. L., Russell, C. T., Gosling, J. T., Bame, S. J., Paschmann, G., Parks, G. K., Anderson, K. A., Anderson, R. R., and Gurnett, D. A.: A macroscopic profile of the typical quasi-perpendicular bow shock - Isee 1 and 2, J. Geophys. Res., 85, 2124-2130, 1980.

Gurevich, A. V. and Pitayevsky, L. P.: Nonstationary structure of a collisionless shock wave, Sov. Phys. JETP-USSR, 38, 291-297, 1974.

Hada, T., Kennel, C. F., and Buti, B.: Stationary nonlinear Alfvén waves and solitons, J. Geophys. Res., 94, 65-77, 1989.

Hobara, Y., Balikhin, M., Krasnoselskikh, V., Gedalin, M., and Yamagishi, H.: Statistical study of the quasi-perpendicular shock ramp widths, J. Geophys. Res., 115, A11106, doi:10.1029/2010JA015659, 2010.

Hong, S. E., Ryu, D., Kang, H. and Cen, R.: Shock waves and cosmic ray acceleration in the outskirts of galaxy clusters, Astrophys. J., 785, 133, doi:10.1088/0004-637X/785/2/133, 2014.

Kennel, C. F.: Shock structure in classical magnetohydrodynamics, J. Geophys. Res., 93, 8545-8857, 1988.

Kennel, C. F. and Sagdeev, R. Z.: Collisionless shock waves in high $\beta$ plasmas, 1, J. Geophys. Res., 72, 3303-3326, 1967.

Kennel, C. F., Edmiston, J. P., Russell, C. T., Scarf, F. L., Coroniti, F. V., Smith, E. J., Tsurutani, B. T., Scudder, J. D., Feldman, W. C. and Anderson, R. R.: Structure of the November 12, 1978, quasi-parallel interplanetary shock, J. Geophys. Res., 89, 54365452, 1984.

Kennel, C. F., Edmiston, J. P., and Hada, T.: A quarter century of collisionless shock research, in: Collisionless shocks in the heliosphere: A tutorial review, vol. 34, pp. 1-36, American Geophysical Union, 1985.

Kennel, C. F., Buti, B., Hada, T., and Pellat, R.: Nonlinear, dispersive, elliptically polarized Alfvén waves, Phys. Fluids, 31, 19491961, 1988.

Krall, N. A. and Trivelpiece, A. W.: Principles of plasma physics, McGraw-Hill, New York, USA, 1973.
Krasnoselskikh, V., Balikhin, M., Walker, S. N., Schwartz, S., Sundkvist, D., Lobzin, V., Gedalin, M., Bale, S. D., Mozer, F., Soucek, J., Hobara, Y., and Comişel, H.: The dynamic quasiperpendicular shock: Cluster discoveries, Space Sci. Rev., 178, 535-598, 2013.

Mellott, M. M.: Subcritical collisionless shock waves, in: Collisionless shocks in the heliosphere: Reviews of current research, 131140, American Geophysical Union, 1985.

Mellott, M. M. and Greenstadt, E. W.: The structure of oblique subcritical bow shocks - ISEE 1 and 2 observations, J. Geophys. Res., 89, 2151-2161, 1984.

Mellott, M. M. and Livesey, W. A.: Shock overshoots revisited, J. Geophys. Res., 92, 13661-13665, 1987.

Neugebauer, M.: Propagating shocks, Space Sci. Rev., 176, 125 132, doi:10.1007/s11214-010-9707-2, 2013.

Ofman, L. and Gedalin, M.: Two-dimensional hybrid simulations of quasi-perpendicular collisionless shock dynamics: Gyrating downstream ion distributions, J. Geophys. Res., 118, 1828-1836, doi:10.1029/2012JA018188, 2013.

Ofman, L., Balikhin, M., Russell, C. T., and Gedalin, M.: Collisionless relaxation of ion distributions downstream of laminar quasi-perpendicular shocks, J. Geophys. Res., 114, A09106, doi:10.1029/2009JA014365, 2009.

Omidi, N., Quest, K. B., and Winske, D.: Low Mach number parallel and quasi-parallel shocks, J. Geophys. Res., 95, 2071720730, 1990.

Papadopoulos, K.: Microinstabilities and anomalous transport, in: Collisionless shocks in the heliosphere: A tutorial review, pp. 5990, American Geophysical Union, 1985.

Russell, C. T., Hoppe, M. M., and Livesey, W. A.: Overshoots in planetary bow shocks, Nature, 296, 45-48, 1982.

Russell, C. T., Jian, L. K., Blanco-Cano, X., and Luhmann, J. G.: STEREO observations of upstream and downstream waves at low Mach number shocks, Geophys. Res. Lett., 36, L03106, doi:10.1029/2008GL036991, 2009.

Ryu, D., Kang, H., Hallman, E. and Jones, T. W.: Cosmological shock waves and their role in the large-scale structure of the Universe. Astrophys. J., 593, 599-610, 2003.

Sagdeev, R. Z.: Cooperative phenomena and shock waves in collisionless plasmas, Rev. Plasma Phys., 4, 23, 1966.

Sagdeev, R. Z. and Galeev, A. A.: Nonlinear Plasma Theory, New York, Benjamin, 1969.

Scudder, J. D., Aggson, T. L., Mangeney, A., Lacombe, C., and Harvey, C. C.: The resolved layer of a collisionless, high beta, supercritical, quasi-perpendicular shock wave. I - RankineHugoniot geometry, currents, and stationarity, J. Geophys. Res., 91, 11019-11052, 1986.

Thomsen, M. F., Gosling, J. T., Bame, S. J., and Mellott, M. M.: Ion and electron heating at collisionless shocks near the critical Mach number, J. Geophys. Res., 90, 137-148, 1985. 